\title{
Photoacoustic image-guided needle biopsy of sentinel lymph nodes
}

Chulhong Kim, Todd N. Erpelding, Walter J. Akers, Konstantin Maslov, Liang Song, et al.

Chulhong Kim, Todd N. Erpelding, Walter J. Akers, Konstantin Maslov, Liang Song, Ladislav Jankovic, Julie A. Margenthaler, Samuel Achilefu, Lihong V. Wang, "Photoacoustic image-guided needle biopsy of sentinel lymph nodes," Proc. SPIE 7899, Photons Plus Ultrasound: Imaging and Sensing 2011, 78990K (17 February 2011); doi: 10.1117/12.874956

SPIE. Event: SPIE BiOS, 2011, San Francisco, California, United States 


\title{
Photoacoustic image-guided needle biopsy of sentinel lymph nodes
}

\author{
Chulhong Kim ${ }^{1}$, Todd N. Erpelding ${ }^{2}$, Walter J. Akers ${ }^{3}$, Konstantin Maslov ${ }^{1}$, Liang Song ${ }^{1}$, Ladislav \\ Jankovic $^{2}$, Julie A. Margenthaler ${ }^{4}$, Samuel Achilefu ${ }^{3}$, and Lihong V. Wang ${ }^{1 *}$ \\ ${ }^{1}$ Optical Imaging Laboratory, Department of Biomedical Engineering, Washington University in St. \\ Louis, Campus Box 1097, One Brookings Dr. St. Louis, Missouri, 63130 \\ ${ }^{2}$ Philips Research North America, 345 Scarborough Road, Briarcliff Manor, NY 10510 \\ ${ }^{3}$ Optical Radiology Laboratory, Department of Radiology and Department of Biophysics, \\ Washington University School of Medicine, 4525 Scott Avenue, Saint Louis, MO 63108 \\ ${ }^{4}$ Department of Surgery, Washington University School of Medicine, \\ Campus Box 8109, 660 South Euclid Avenue, St. Louis, Missouri 63110
}

\begin{abstract}
We have implemented a hand-held photoacoustic and ultrasound probe for image-guided needle biopsy using a modified clinical ultrasound array system. Pulsed laser light was delivered via bifurcated optical fiber bundles integrated with the hand-held ultrasound probe. We photoacoustically guided needle insertion into rat sentinel lymph nodes (SLNs) following accumulation of indocyanine green (ICG). Strong photoacoustic image contrast of the needle was achieved. After intradermal injection of ICG in the left forepaw, deeply positioned SLNs (beneath 2-cm thick chicken breast) were easily indentified in vivo and in real time. Further, we confirmed ICG uptake in axillary lymph nodes with in vivo and ex vivo fluorescence imaging. These results demonstrate the clinical potential of this hand-held photoacoustic system for facile identification and needle biopsy of SLNs for cancer staging and metastasis detection in humans.
\end{abstract}

Keywords: Image-guided needle biopsy, photoacoustic imaging, sentinel lymph node biopsy.

\section{INTRODUCTION}

The surgical removal of primary breast tumor and dissection of all level I and level II axillary lymph nodes were widely performed in the treatment of invasive breast cancers. ${ }^{1}$ However, common side effects after axillary lymph node dissection (ALND) include upper-extremity lymphedema, arm numbness, impaired shoulder mobility, arm weakness, and infections in the breast, chest, or arm. ${ }^{2,3}$ Therefore, as a less invasive alternative to ALND, sentinel lymph node biopsy (SLNB), that is, biopsy of the first lymph node receiving drainage from a cancer-containing area of the breast, has become increasingly important in breast cancer staging and treatment. ${ }^{4}$ By reducing the number of lymph nodes removed, SLNB reduces the risk of side effects compared to ALND. ${ }^{2}$ Although SLNB with methylene blue dye and radioactive tracers has an identification rate of $90-95 \%$ and a sensitivity of $88-95 \%,{ }^{2-4}$ it remains a highly invasive procedure involving ionizing radiation.

Photoacoustic (PA) imaging is a nonionizing and noninvasive hybrid imaging technique that combines strong optical absorption contrast with high ultrasonic spatial resolution. ${ }^{5,6}$ Since ultrasonic scattering is $2-3$ orders of magnitude less than optical scattering, PA imaging can break through the fundamental depth limitations of pure optical imaging. Using intrinsic contrasts such as hemoglobin or melanin, PA imaging can provide both morphological and functional information. Moreover, using molecularly targeted exogenous contrast agents, it can also provide molecular

*Corresponding author: lhwang@biomed.wustl.edu

Photons Plus Ultrasound: Imaging and Sensing 2011, edited by Alexander A. Oraevsky, Lihong V. Wang, Proc. of SPIE Vol. 7899, 78990K · (C) 2011 SPIE · CCC code: 1605-7422/11/\$18 · doi: 10.1117/12.874956 
information. We have previously reported in vivo detection of lymph nodes in rats using a raster-scanning table-top PA imaging system. Methylene blue, indocyanine green (ICG), Evans blue, and gold nanoparticles were used as contrast agents. ${ }^{711}$ However, this system was relatively slow for clinical applications $\left(20\right.$ minutes for a $20 \times 20 \mathrm{~mm}^{2}$ field of view), and its imaging head was not hand-held. We have also reported in vivo PA and US mapping of SLNs in rats using a US array, but the light delivery was not hand-held. ${ }^{11}$

In this proceedings article, we report noninvasive in vivo mapping of deeply positioned ICG-dyed SLNs $(\sim 2.1 \mathrm{~cm})$ in rats using a PA imaging system adapted from a commercial US array system. An US probe integrated with optical fiber bundles for light delivery enabled hand-held scanning as in ultrasonography. ICG uptake in SLNs was confirmed by fluorescence imaging. Furthermore, real-time PA imaging (1 frame per second (fps)) successfully guided needle insertion.

\section{METHODS AND MATERIALS}

Figure 1 describes the schematic of the combined PA and US imaging system, adapted from a clinical US imaging system (iU22, Philips Healthcare). Raw per-channel PA and US data were both acquired through the modified channel board architecture and transferred to a custom-made data acquisition computer for display and post-processing. Laser firing was synchronized with the data acquisition system. Fourier beam formed PA images were displayed at a frame rate of $\sim 1$ fps. US images were captured at 5 fps. For this study, we used a linear array US probe (L8-4, Philips Healthcare) with a nominal bandwidth of 4-8 MHz. Light from a tunable dye laser (PrecisionScan-P, Sirah), pumped by a Q-switched Nd:YAG laser (PRO-350-10, Newport) with a 6.5-ns pulse duration and a 10-Hz pulse repetition rate, was coupled to a multimode fiber bundle (CB18043, Fiberguide). The bifurcated fiber bundle was physically integrated with the US probe enabling hand-held scanning. The two optical beams were obliquely incident $\left(\sim 45^{\circ}\right)$ on the tissue surface as rectangles $(2 \mathrm{~cm} \times 3 \mathrm{~cm}$ along the $X$ and $Y$ axes from each bundle). An optical wavelength of $810 \mathrm{~nm}$, close to the peak optical absorption wavelength of ICG (806 nm in albumin), was utilized. Light fluence on the skin was less than $1.5 \mathrm{~mJ} / \mathrm{cm}^{2}$, well below the American National Standards Institute safety limits. ${ }^{12}$

Animal handling was performed according to the guidelines on the care and use of laboratory animals at Washington University in St. Louis. Sprague Dawley rats $(\sim 200 \mathrm{~g})$ were initially anesthetized using a mixture of ketamine (85 $\mathrm{mg} / \mathrm{kg}$ ) and xylazine $(15 \mathrm{mg} / \mathrm{kg}$ ). We layered $\sim 2 \mathrm{~cm}$ of chicken tissue atop the rat to increase the penetration depth. First, a control PA image was acquired before the injection of ICG. After intradermally injecting $0.1 \mathrm{ml}$ of 1-mM ICG into the left forepaw, a series of PA images were obtained to monitor the ICG uptake in the SLN dynamically. After PA imaging, in vivo fluorescence imaging was performed with a time-domain diffuse optical imaging system (eXplore Optix, Advance Research Technologies) to confirm ICG uptake. Then, we demonstrated PA image-guided needle (18 gauge) insertion toward a ICG-stained lymph node.

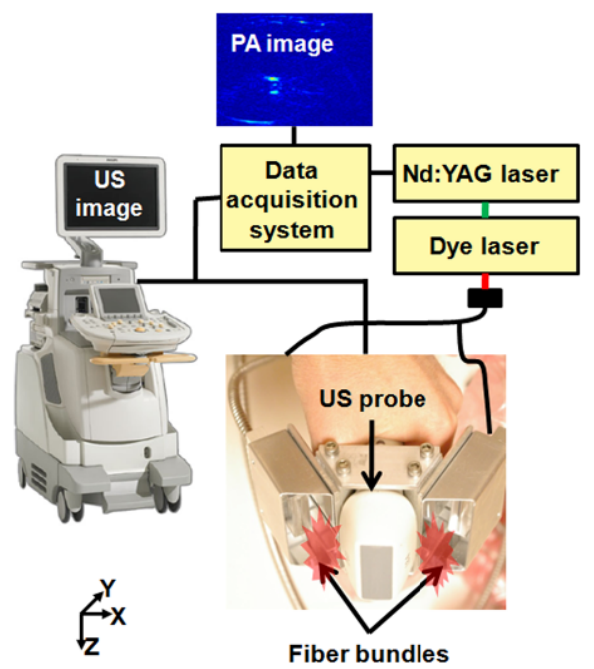

Figure 1: Experimental setup of the combined photoacoustic (PA) and ultrasound (US) imaging probe based on a clinical ultrasound scanner (iU22, Philips Healthcare). 


\section{RESULTS AND DISCUSSION}

We investigated the feasibility of PA and US mapping of SLNs with ICG. The left axillary region in a rat was imaged after ICG injection. Figure 2, created by overlaying the PA and US images, shows both morphological information and functional information (ICG uptake in the SLN). The PA image was displayed with pseudocolor, while the US image was with gray color. 2-cm deep rat SLN was clearly identified in the integrated PA and US image.

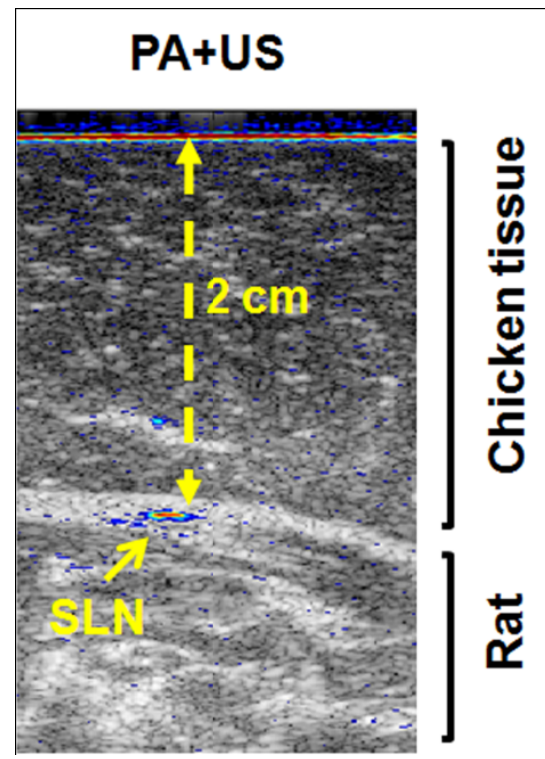

Figure 2: In vivo PA and US B-scan imaging of a SLN dyed with ICG. Both PA (pseudo color) and US (gray scale) images were overlaid.

Fluorescence images obtained after PA imaging confirmed ICG uptake in the SLN (Fig. 3). Only the first draining node, the sentinel node, generated strong fluorescence.

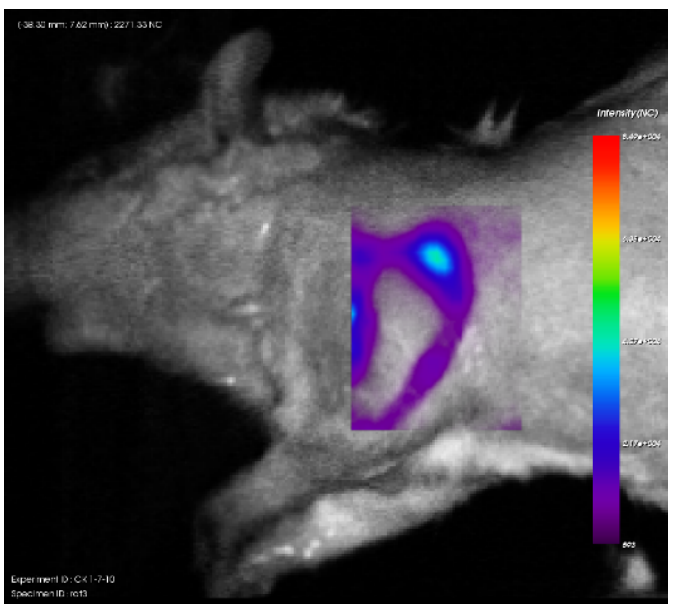

Figure 3: In vivo fluorescence imaging of a rat SLN dyed with ICG.

The needle progression could also be detected photoacoustically. As shown in Fig. 4. PA imaging was able to detect the SLN and the needle, clearly. Unlike US imaging, PA imaging does not offer any speckle artifacts, consequently the PA image contrast is much higher than the US image contrast. In addition, ultrasound imaging has a limited angular sensitivity for detecting the needle, as most of the incident acoustic energy is reflected away from the limited aperture 
ultrasound probe. In comparison, photoacoustic waves generated following optical absorption and thermoelastic expansion of are omni-directional. As a result, photoacoustic imaging offers an improved angular sensitivity compared with conventional ultrasound imaging.

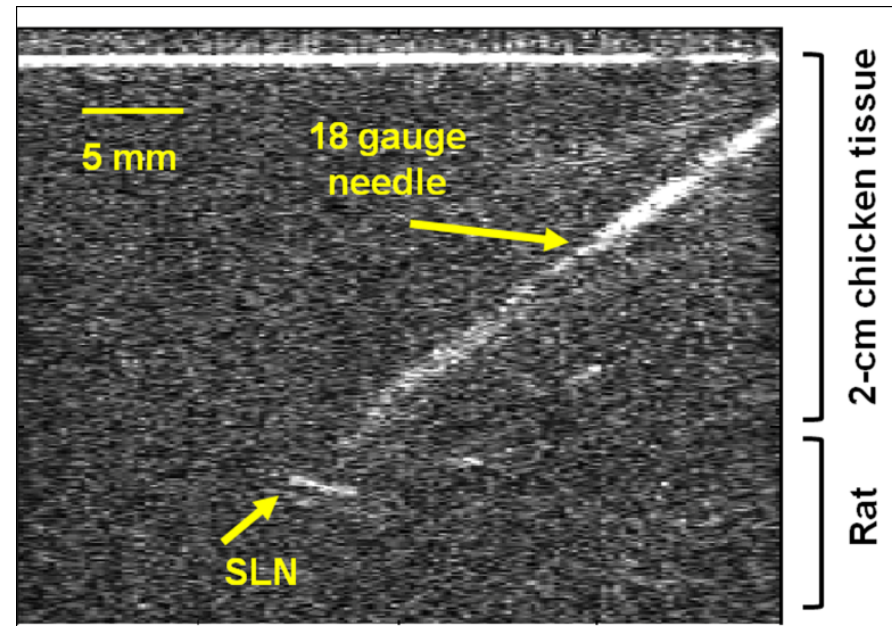

Figure 4: In vivo photoacoustic (PA) and ultrasound (US) B-scan imaging of a sentinel lymph node (SLN) dyed with indocyanine green (ICG) and guiding of a biopsy needle.

The development of a real-time, noninvasive technique for identifying sentinel lymph nodes offers the possibility of percutaneous needle biopsies for axillary staging of breast cancer. Percutaneous needle biopsies would offer patients a nonsurgical option that is not currently available, likely improving patient outcomes while reducing patient morbidity. Currently, the results for SLNB performed during lumpectomy are often not known until after surgery, so for positive SLNB patients a second surgical visit to undergo ALND is required. Needle biopsies could be performed preoperatively and, in cases of positive lymph nodes, ALND can be performed at the time of lumpectomy, without needing a second surgical visit. Photoacoustic imaging has the unique capability to image both the needle and sentinel lymph nodes stained by optical dyes, which provides a strong clinical potential for this technique. Photoacoustic imaging is compatible with clinically used dyes such as ICG, as demonstrated here, and methylene blue, which is more commonly used during SLNB.

\section{CONCLUSIONS}

We have developed a noninvasive, nonionizing, real-time, and hand-held PA and US imaging system using a modified clinical US imaging system. In vivo mapping of rat SLNs at an imaging depth of $\sim 2.1 \mathrm{~cm}$ was successfully accomplished following ICG injection. Further, PA imaging enabled guided needle insertion with strong contrast in a tissue mimicking phantom. This technique is highly translatable to clinical applications for image-guided SLN biopsy, nerve blockings, and many others.

\section{ACKNOWLEDGEMENT}

This work was supported in part by grants from National Institutes of Health (R01 EB000712, R01 EB008085, R01 NS46214, and U54 CA136398 -- the Network for Translational Research -- to L.V.W.). L.V.W. has a financial interest in Endra, Inc., which, however, did not support this work. 


\section{REFERENCES}

[1] H. Kobayashi, S. Kawamoto, Y. Sakai, et al., "Lymphatic drainage imaging of breast cancer in mice by micromagnetic resonance lymphangiography using a nano-size paramagnetic contrast agent," J. Natl. Cancer. I. 96(9), 703708 (2004).

[2] K. M. McMasters, T. M. Tuttle, D. J. Carlson, et al., "Sentinel lymph node biopsy for breast cancer: a suitable alternative to routine axillary dissection in multi-institutional practice when optimal technique is used," J. Clin. Oncol., 18(13), 2560-2566 (2000).

[3] O. A. Ung, "Australasian experience and trials in sentinel lymph node biopsy: the RACS SNAC trial," Asian. J. Surg. 27(4), 284-290 (2004).

[4] D. Krag, D. Weaver, T. Ashikaga, et al., "The sentinel node in breast cancer - a multicenter validation study," N. Engl. J. Med., 339, 941 (1998).

[5] C. Kim, C. Favazza and L. V. Wang, "In vivo photoacoustic tomography of chemicals: high-resolution functional and molecular optical imaging at new pepths," Chem. Rev. 110(5), 2756-2782(2010).

[6] H. F. Zhang, K. Maslov, G. Stoica, et al., "Functional photoacoustic microscopy for high-resolution and noninvasive in vivo imaging," Nat. Biotechnol. 24(7), 848-851 (2006).

[7] K. H. Song, E. W. Stein, J. A. Margenthaler, et al., "Noninvasive photoacoustic identification of sentinel lymph nodes containing methylene blue in vivo in a rat model," J. Biomed. Opt. 13(5), 054033 (2008).

[8] K. H. Song, C. Kim, C. M. Cobley, et al., "Near-infrared gold nanocages as a new class of tracers for photoacoustic sentinel lymph node mapping on a rat model," Nano Lett. 9(1), 183-188 (2009).

[9] L. Song, C. Kim, K. Maslov, et al., "High-speed dynamic 3-D photoacoustic imaging of sentinel lymph node in a murine model using an ultrasound array," Med. Phys. 36(8), 3724-3729 (2009).

[10] C. Kim, K. H. Song, F. Gao, and L. V. Wang, " Sentinel lymph nodes and lymphatic vessels: noninvasive dualmodality in vivo mapping by using indocyanine green in rats-volumetric spectroscopic photoacoustic imaging and planar fluorescence imaging," Radiology 255, 442-450 (2010).

[11] T. N. Erpelding, C. Kim, M. Pramanik, et al., "Sentinel lymph nodes in the rat: noninvasive photoacoustic and US imaging with a clinical US system," Radiology 256(1), 102-110 (2010).

[12] American national standard for the safe use of lasers, Standard Z136.1-2000 (ANSI. Inc, New York, 2002). 